\title{
A versatile, refrigerant- and cryogen-free cryofocusing-thermodesorption unit for preconcentration of traces gases in air
}

Florian Obersteiner et al.

Correspondence to: Florian Obersteiner (obersteiner@iau.uni-frankfurt.de)

The copyright of individual parts of the supplement might differ from the CC-BY 3.0 licence. 


\section{Supplementary Information}

2 Table S-1 shows a list of substances detected up to the time of completion of this paper. Iden-

3 tifications based on ambient air samples as well as synthetic mixtures. Substances are separat-

4 ed into six classes (e.g. CFCs and HCFCs, PFCs and HFCs etc.), which are listed in arbitrary

5 order. Within each class, substances are sorted according to their boiling point (bp) in $\left[{ }^{\circ} \mathrm{C}\right]$.

6 Chemical sum formula as well as retention time $t_{R}$ in [min] on the GS GasPro PLOT column

7 listed in columns two and three. Columns $5 \& 6$ show analyte residues in [\%], expressed as

8 chromatographic signal area determined in a blank gas measurement relative to a signal area

9 determined in a preceding $1 \mathrm{~L}$ ambient air sample. Blank gas: purified helium 6.0 (Praxair,

10 Germany). "Residue HayeSep D" denotes residues found with HayeSep D as adsorptive mate-

11 rial, "Residue Unibeads $1 \mathrm{~S}$ " shows the same for Unibeads $1 \mathrm{~S}$ as adsorptive material. Residues

12 that a constant background (contamination), are marked with a " $c$ ", ones that represent a

13 memory effect from a preceding sample are marked with an " $m$ ". Substances that are not de-

14 tected regularly in ambient air samples or show poor measurement precision $\geq 10 \%$ were 15 excluded from the analysis ("not analysed"; n.a.). If no residue was detected or the detected 16 residue was $\leq 0.01 \%$, a "not detected" (n.d.) is assigned to the respective substance.

17 Table S-1. List of detectable substances and blank residues. Descriptions are given in the text.

\begin{tabular}{|c|c|c|c|c|c|}
\hline Class/Name & Formula & $t_{R}[\min ]$ & bp $\left[{ }^{\circ} \mathrm{C}\right]$ & $\begin{array}{l}\text { Residue } \\
\text { HayeSep D }\end{array}$ & $\begin{array}{l}\text { Resi } \\
\text { Unib }\end{array}$ \\
\hline \multicolumn{6}{|c|}{ CFCs \& HCFCs } \\
\hline HCFC-22 & $\mathrm{CHClF}_{2}$ & 5.20 & -41 & n.d. & n.d. \\
\hline CFC-115 & $\mathrm{CCIF}_{2} \mathrm{CF}_{3}$ & 4.48 & -39 & n.d. & n.o \\
\hline CFC-12 & $\mathrm{CF}_{2} \mathrm{Cl}_{2}$ & 5.02 & -30 & n.d. & n.d \\
\hline HCFC-124 & $\mathrm{CHF}_{2} \mathrm{CF}_{2} \mathrm{Cl}$ & 6.85 & -12 & n.d. & n.o \\
\hline HCFC-142b & $\mathrm{CH}_{3} \mathrm{CCIF}_{2}$ & 6.87 & -10 & n.d. & n.d \\
\hline HCFC-31 & $\mathrm{CH}_{2} \mathrm{ClF}$ & 6.40 & -9 & n.a. & 11. \\
\hline CFC-114 & $\mathrm{CCIF}_{2} \mathrm{CCIF}_{2}$ & 6.67 & 4 & n.d. & n.d \\
\hline HCFC-133a & $\mathrm{C}_{2} \mathrm{H}_{2} \mathrm{ClF}_{3}$ & 7.55 & 6 & n.d. & n.d. \\
\hline HCFC-21 & $\mathrm{CHFCl}_{2}$ & 7.32 & 9 & n.d. & II. \\
\hline CFC-11 & $\mathrm{CFCl}_{3}$ & 7.28 & 24 & n.d. & n.c \\
\hline HCFC-141b & $\mathrm{CH}_{3} \mathrm{CCl}_{2} \mathrm{~F}$ & 8.42 & 32 & n.d. & n.d. \\
\hline HCFC-1121 & $\mathrm{CHCICFCl}$ & 8.05 & 35 & n.a. & $\mathrm{n}$. \\
\hline
\end{tabular}




\begin{tabular}{llllll}
\hline Class/Name & Formula & $\mathbf{t}_{\mathbf{R}}$ [min] & $\mathbf{b p}\left[{ }^{\circ} \mathbf{C}\right.$ ] & $\begin{array}{l}\text { Residue } \\
\text { HayeSep } \mathbf{D}\end{array}$ & $\begin{array}{l}\text { Residue } \\
\text { Unibeads 1S }\end{array}$ \\
\hline HCFC-132b & $\mathrm{CH}_{2} \mathrm{ClCClF}_{2}$ & 9.08 & 46 & n.d. & n.d. \\
CFC-113 & $\mathrm{CCl}_{2} \mathrm{FCClF}_{2}$ & 8.45 & 48 & $0.2 \%(\mathrm{~m})$ & n.d. \\
HCFC-225ca & $\mathrm{CF}_{3} \mathrm{CF}_{2} \mathrm{CHCl}_{2}$ & 9.37 & 51 & n.a. & n.a. \\
HCFC-225cb & $\mathrm{CClF}_{2} \mathrm{CF}_{2} \mathrm{CHCIF}$ & 9.57 & 56 & n.a. & n.a. \\
CFC-112 & $\mathrm{CFCl}_{2} \mathrm{CFCl}_{2}$ & 10.33 & 92 & n.d. & n.d. \\
HCFC-131 & $\mathrm{CCl}_{3} \mathrm{CH}_{2} \mathrm{~F}$ & 12.38 & 103 & n.a. & n.a.
\end{tabular}

\section{PFCs \& HFCs}

\begin{tabular}{|c|c|c|c|c|c|}
\hline HFC-23 & $\mathrm{CHF}_{3}$ & 3.01 & -82 & $2.6 \%(c)$ & n.a. \\
\hline HFC-41 & $\mathrm{CH}_{3} \mathrm{~F}$ & 4.38 & -78 & n.a. & n.a. \\
\hline HFC-32 & $\mathrm{CH}_{2} \mathrm{~F}_{2}$ & 4.20 & -52 & n.d. & n.d. \\
\hline HFC-125 & $\mathrm{CHF}_{2} \mathrm{CF}_{3}$ & 4.87 & -49 & $0.4 \%(c)$ & $1.3 \%(\mathrm{c})$ \\
\hline HFC-143a & $\mathrm{CH}_{3} \mathrm{CF}_{3}$ & 5.00 & -48 & n.d. & n.d. \\
\hline HFC-161 & $\mathrm{C}_{2} \mathrm{H}_{5} \mathrm{~F}$ & 6.85 & -38 & n.a. & n.a. \\
\hline PFC-218 & $\mathrm{C}_{3} \mathrm{~F}_{8}$ & 4.02 & -37 & n.d. & n.d. \\
\hline PFC-216 & $\mathrm{C}_{3} \mathrm{~F}_{6}$ & 4.58 & -30 & n.a. & n.a. \\
\hline HFO-1234yf & $\mathrm{CHFCHCF}_{3}$ & 5.72 & -28 & $6.9 \%(c)$ & $14.9 \%$ (c) \\
\hline HFC-134a & $\mathrm{CH}_{2} \mathrm{FCF}_{3}$ & 5.92 & -26 & n.d. & n.d. \\
\hline HFC-152a & $\mathrm{CH}_{3} \mathrm{CHF}_{2}$ & 6.53 & -25 & n.d. & n.d. \\
\hline HFC-134 & $\mathrm{CHF}_{2} \mathrm{CHF}_{2}$ & 6.32 & -23 & $1.1 \%(c)$ & $3.0 \%(c)$ \\
\hline HFC-227ea & $\mathrm{CF}_{3} \mathrm{CHFCF}_{3}$ & 6.52 & -16 & n.d. & n.d. \\
\hline HFO-1234ze & $\mathrm{CHFCHCF}_{3}$ & 6.27 & -16 & n.d. & n.d. \\
\hline PFC-318 & $c-\mathrm{C}_{4} \mathrm{~F}_{8}$ & 5.68 & -6 & n.d. & n.d. \\
\hline HFC-236fa & $\mathrm{CF}_{3} \mathrm{CH}_{2} \mathrm{CF}_{3}$ & 7.22 & -1 & n.d. & n.d. \\
\hline HFC-329ccb & $\mathrm{C}_{4} \mathrm{HF}_{9}$ & 7.67 & 15 & n.a. & n.a. \\
\hline HFC-245fa & $\mathrm{CF}_{3} \mathrm{CH}_{2} \mathrm{CHF}_{2}$ & 7.92 & 15 & n.d. & n.d. \\
\hline HFO-1233zd & $\mathrm{CHClCHCF}_{3}$ & 7.82 & 19 & n.a. & n.a. \\
\hline HFC-356mff & $\mathrm{C}_{4} \mathrm{H}_{4} \mathrm{~F}_{6}$ & 8.35 & 25 & n.a. & n.a. \\
\hline HFC-365mfc & $\mathrm{CF}_{3} \mathrm{CH}_{2} \mathrm{CF}_{2} \mathrm{CH}_{3}$ & 9.27 & 40 & n.a. & n.a. \\
\hline \multicolumn{6}{|l|}{ Halons } \\
\hline Halon-1301 & $\mathrm{CBrF}_{3}$ & 3.87 & -58 & n.d. & n.d. \\
\hline Halon-1211 & $\mathrm{CBrClF}_{2}$ & 6.32 & -4 & n.d. & n.d. \\
\hline Halon-1202 & $\mathrm{CF}_{2} \mathrm{Br}_{2}$ & 7.45 & 23 & n.a. & n.a. \\
\hline Halon-2402 & $\mathrm{CBrF}_{2} \mathrm{CBrF}_{2}$ & 8.53 & 47 & n.d. & n.d. \\
\hline Halon-2311 & $\mathrm{CF}_{3} \mathrm{CHBrCl}$ & 9.30 & 50 & n.a. & n.a. \\
\hline
\end{tabular}




\begin{tabular}{|c|c|c|c|c|}
\hline Class/Name & Formula & $t_{R}[\min ]$ bp $\left[{ }^{\circ} \mathrm{C}\right]$ & $\begin{array}{l}\text { Residue } \\
\text { HayeSep D }\end{array}$ & $\begin{array}{l}\text { Residue } \\
\text { Unibeads 1S }\end{array}$ \\
\hline
\end{tabular}

\section{Chloro-, Bromo- \& lodocarbons}

\begin{tabular}{|c|c|c|c|c|c|}
\hline Chloromethane & $\mathrm{CH}_{3} \mathrm{Cl}$ & 6.02 & -24 & $0.5 \%(c)$ & $0.6 \%(c)$ \\
\hline Bromomethane & $\mathrm{CH}_{3} \mathrm{Br}$ & 7.00 & 4 & $3.4 \%(c)$ & $1.8 \%(c)$ \\
\hline Chloroethane & $\mathrm{C}_{2} \mathrm{H}_{5} \mathrm{Cl}$ & 7.92 & 12 & $25.5 \%(c)$ & $8.6 \%(c)$ \\
\hline Dichloromethane & $\mathrm{CH}_{2} \mathrm{Cl}_{2}$ & 8.17 & 40 & $0.4 \%(c, m)$ & $0.2 \%(c)$ \\
\hline lodomethane & $\mathrm{CH}_{3} \mathrm{I}$ & 8.00 & 42 & $43.9 \%(c, m)$ & $46.2 \%(c, m)$ \\
\hline Trichloromethane & $\mathrm{CHCl}_{3}$ & 8.92 & 61 & $1.4 \%(c, m)$ & $0.7 \%(c, m)$ \\
\hline Bromochloromethane & $\mathrm{CH}_{2} \mathrm{BrCl}$ & 9.03 & 68 & n.d. & n.d. \\
\hline Methyl chloroform & $\mathrm{CH}_{3} \mathrm{CCl}_{3}$ & 9.93 & 74 & n.d. & n.d. \\
\hline Tetrachloromethane & $\mathrm{CCl}_{4}$ & 9.08 & 77 & $1.1 \%(\mathrm{~m})$ & n.d. \\
\hline Trichloroethene & $\mathrm{C}_{2} \mathrm{HCl}_{3}$ & 9.55 & 87 & n.d. & n.d. \\
\hline Bromodichloromethane & $\mathrm{CHBrCl}_{2}$ & 10.10 & 90 & n.d. & n.d. \\
\hline Dibromomethane & $\mathrm{CH}_{2} \mathrm{Br}_{2}$ & 10.03 & 96 & n.d. & n.d. \\
\hline Dibromochloromethane & $\mathrm{CHBr}_{2} \mathrm{Cl}$ & 11.53 & 119 & n.d. & n.d. \\
\hline Tetrachloroethene & $\mathrm{C}_{2} \mathrm{Cl}_{4}$ & 10.62 & 121 & $23.9 \%(c, m)$ & $5.2 \%(c, m)$ \\
\hline Tribromomethane & $\mathrm{CHBr}_{3}$ & 13.50 & 147 & $11.2 \%(m)$ & n.d. \\
\hline Diiodomethane & $\mathrm{CH}_{2} \mathrm{I}_{2}$ & 15.00 & 181 & n.a. & n.a. \\
\hline
\end{tabular}

\section{Sulfur-containing and other halogenated compounds}

$\begin{array}{llll}\text { Sulfuryldifluoride } & \mathrm{SO}_{2} \mathrm{~F}_{2} & 4.20 & -55 \\ \text { Carbonyl sulfide } & \mathrm{COS} & 3.77 & -50 \\ \text { Chlorotrifluoroethylene } & \mathrm{C}_{2} \mathrm{~F}_{3} \mathrm{Cl} & 4.92 & -28 \\ \text { Perfluorotetrahydrofuran } & \mathrm{C}_{4} \mathrm{~F}_{8} \mathrm{O} & 5.87 & 2 \\ \text { 3-chloropentafluoropropene } & \mathrm{CF}_{2} \mathrm{CFCF}_{2} \mathrm{Cl} & 8.07 & 8 \\ \text { Desflurane } & \mathrm{CF}_{3} \mathrm{CHFOCHF}_{2} & 8.42 & 24 \\ \text { Carbon disulfide } & \mathrm{CS}_{2} & 6.54 & 46 \\ \text { Isoflurane } & \mathrm{CHF}_{2} \mathrm{OCHClCF}_{3} & 9.83 & 49 \\ \text { Sevoflurane } & \mathrm{CF}_{3} \mathrm{CF}_{3} \mathrm{CHOCH}_{2} \mathrm{~F} & 10.35 & 59\end{array}$

n.d. $\quad$ n.d.

$0.4 \%(c) \quad 0.1 \%(c)$

n.a. n.a.

n.a. n.a.

n.d. $\quad 7.6 \%$ (c)

n.a. n.a.

$4.0 \%(\mathrm{c}) \quad 0.8 \%(\mathrm{c})$

n.a. n.a.

n.a. n.a. 


\begin{tabular}{|c|c|c|c|c|c|}
\hline Class/Name & Formula & $t_{R}[\min ]$ & bp $\left[{ }^{\circ} \mathrm{C}\right]$ & $\begin{array}{l}\text { Residue } \\
\text { HayeSep D }\end{array}$ & $\begin{array}{l}\text { Residue } \\
\text { Unibeads 1S }\end{array}$ \\
\hline \multicolumn{6}{|c|}{ Hydrocarbons and Aldehydes } \\
\hline Ethyne & $\mathrm{C}_{2} \mathrm{H}_{2}$ & 3.75 & -81 & $0.3 \%(c)$ & $1.4 \%(c)$ \\
\hline Propene & $\mathrm{C}_{3} \mathrm{H}_{6}$ & 5.38 & -48 & $35.2 \%(\mathrm{c})$ & $28.5 \%$ (c) \\
\hline Propane & $\mathrm{C}_{3} \mathrm{H}_{8}$ & 4.09 & -42 & $0.4 \%(c)$ & $0.1 \%(c)$ \\
\hline Propyne & $\mathrm{C}_{3} \mathrm{H}_{4}$ & 7.17 & -23 & n.d. & n.d. \\
\hline Formaldehyde & $\mathrm{CH}_{2} \mathrm{O}$ & 7.62 & -19 & n.a. & n.a. \\
\hline Isobutane & $\mathrm{C}_{4} \mathrm{H}_{10}$ & 5.79 & -13 & $0.7 \%(c)$ & $1.0 \%(c)$ \\
\hline Isobutene & $\mathrm{C}_{4} \mathrm{H}_{8}$ & 7.32 & -7 & n.d. & $75.3 \%(c)$ \\
\hline 1-butene & $\mathrm{C}_{4} \mathrm{H}_{8}$ & 7.38 & -6 & n.a. & n.a. \\
\hline 1,3-butadiene & $\mathrm{C}_{4} \mathrm{H}_{6}$ & 7.32 & -4 & n.a. & n.a. \\
\hline n-butane & $\mathrm{C}_{4} \mathrm{H}_{10}$ & 6.05 & -1 & $0.3 \%(\mathrm{c})$ & $0.1 \%(c)$ \\
\hline trans-2-butene & $\mathrm{C}_{4} \mathrm{H}_{8}$ & 7.02 & 1 & $25.3 \%(c)$ & $19.8 \%(c)$ \\
\hline cis-2-butene & $\mathrm{C}_{4} \mathrm{H}_{8}$ & 7.24 & 4 & n.a. & n.a. \\
\hline Acetaldehyde & $\mathrm{C}_{2} \mathrm{H}_{4} \mathrm{O}$ & 11.26 & 20 & $99.2 \%(c, m)$ & $82.0 \%(c, m)$ \\
\hline 2-methylbutane & $\mathrm{C}_{5} \mathrm{H}_{10}$ & 7.40 & 28 & $0.4 \%(m)$ & $0.2 \%(\mathrm{~m})$ \\
\hline Isoprene & $\mathrm{C}_{5} \mathrm{H}_{8}$ & 8.67 & 34 & n.a. & n.a. \\
\hline n-pentane & $\mathrm{C}_{5} \mathrm{H}_{12}$ & 7.57 & 36 & $0.7 \%(m)$ & $0.3 \%(m)$ \\
\hline trans-2-pentene & $\mathrm{C}_{5} \mathrm{H}_{10}$ & 8.47 & 36 & n.d. & $22.2 \%(c, m)$ \\
\hline cis-2-pentene & $\mathrm{C}_{5} \mathrm{H}_{10}$ & 8.56 & 37 & n.a. & n.a. \\
\hline 2-methylpentane & $\mathrm{C}_{6} \mathrm{H}_{14}$ & 8.61 & 60 & $0.8 \%(\mathrm{~m})$ & $1.0 \%(\mathrm{~m})$ \\
\hline 3-methylpentane & $\mathrm{C}_{6} \mathrm{H}_{14}$ & 8.71 & 63 & $1.8 \%(\mathrm{~m})$ & n.d. \\
\hline n-hexane & $\mathrm{C}_{6} \mathrm{H}_{14}$ & 8.71 & 68 & $1.5 \%(\mathrm{c})$ & n.d. \\
\hline Benzene & $\mathrm{C}_{6} \mathrm{H}_{6}$ & 11.00 & 80 & $2.5 \%(c)$ & $5.2 \%(c)$ \\
\hline Cyclohexane & c- $\mathrm{C}_{6} \mathrm{H}_{12}$ & 8.82 & 81 & n.d. & n.d. \\
\hline n-heptane & $\mathrm{C}_{7} \mathrm{H}_{16}$ & 10.06 & 98 & $23.1 \%(\mathrm{c}, \mathrm{m})$ & $4.0 \%(\mathrm{~m})$ \\
\hline Toluene & $\mathrm{C}_{7} \mathrm{H}_{8}$ & 14.52 & 111 & $17.4 \%(\mathrm{c}, \mathrm{m})$ & $9.8 \%(\mathrm{c}, \mathrm{m})$ \\
\hline
\end{tabular}

